\title{
Shape-based Peak Identity Confirmation in Liquid Chromatography
}

\author{
Akinde F. Kadjo ${ }^{\star a}$, Purnendu K. Dasgupta ${ }^{\mathrm{b}}$, Kannan Srinivasan ${ }^{\mathrm{a}}$ \\ aThermo Fisher Scientific, 1228 Titan way, Building 1, Sunnyvale, CA 94085, USA \\ ${ }^{\mathrm{b} D e p a r t m e n t}$ of Chemistry and Biochemistry, The University of Texas at Arlington, \\ Arlington, TX 76019, USA
}

\section{SUPPORTING INFORMATION Table of Content}

Table S1. See separate SI file Table S1.xlsx

Figure S1. Calibration mapping for fluoride $\quad$...S3

Figure S2. Ability of a third-degree polynomial to predict fluoride peak shape $\quad$...S4

Table S2. Prediction accuracy: Present strategy vs. general gaussian model ...S5

Figure S3. Illustrative good vs. poor match $\quad$...S6

Figure S4. Illustration of a poor match and corresponding regression plot $\quad$...S7

Figure S5. Illustration of index of width mismatch (IWM) _.S8

Figure S6. Graphical IWMn plot for the illustration in Figure S5.

Figure S7. 6 anions standard run on an AS15 column.

Table S3. AS15 Column Correlation Matrix

Figure S8. Chromatogram of a water sample $\quad$...S12

Figure S9. Overlaid chromatograms of Sulfate and Nitrate $\quad . . S 13$

Figure S10. Overlaid chromatograms of dimethyl phthalate and acetophenone ...S14

Table S4. AS18 Column Correlation Matrix $\quad$...S15

Table S5. AS19 Column Correlation Matrix $\quad$...S16

Table S6. AS22 Column Correlation Matrix $\quad$...S17

Table S7. CS16 Column Correlation Matrix $\quad$...S18

Figure S11. Separation of phenylurea pesticides $\quad$...S19

Table S8. Phenylurea Analysis $r^{2}$ Correlation Matrix $\quad \ldots$ S20

${ }^{*}$ Corresponding author. E-mail address: akindeflorence.kadjo@mavs.uta.edu (Akinde Kadjo 


\section{Table S1. Calibration Matrix for Fluoride, AS19 Column}

\section{Complete Unabridged Version of Table 1}

Because of length (over 2000 rows of data, this table appears as a separate Excel file (Table S1.xlsx). 


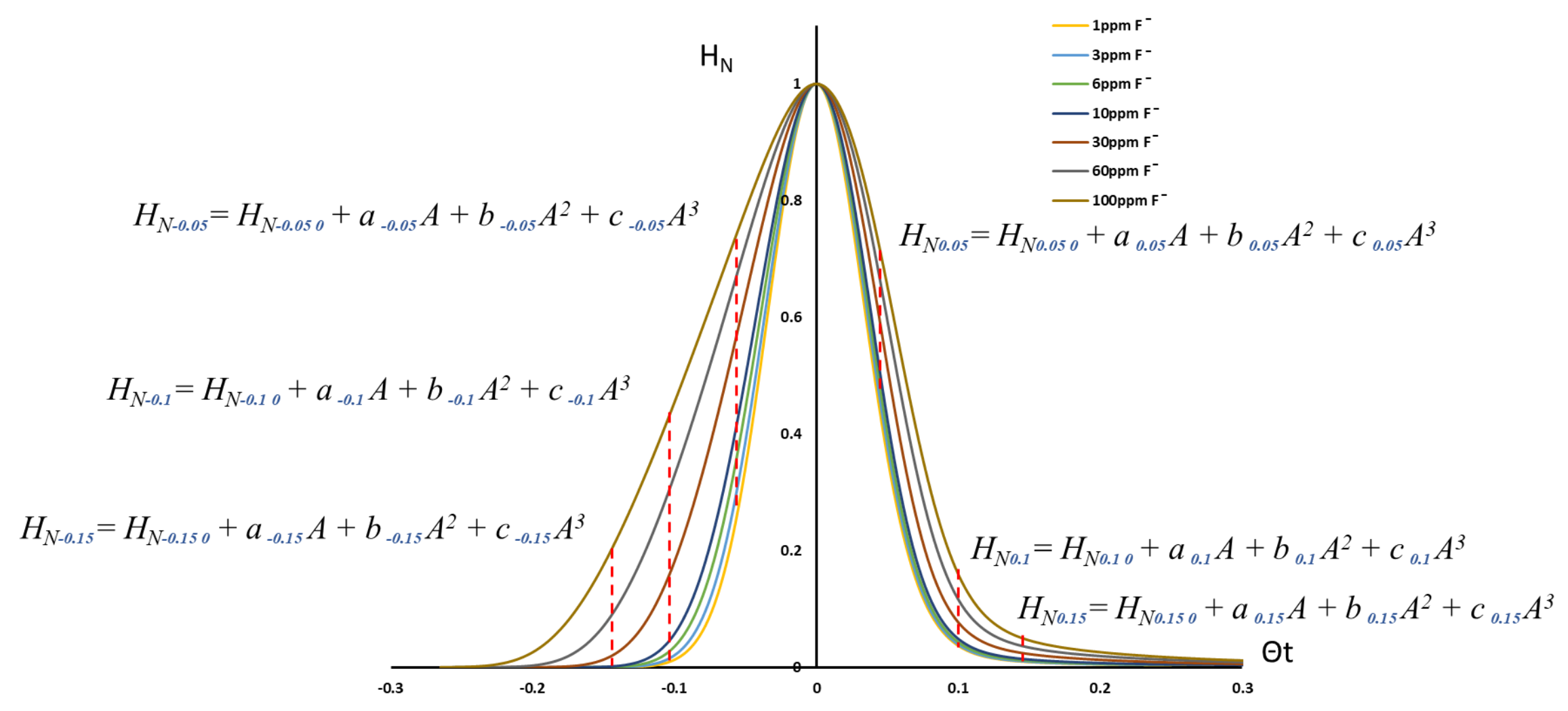

Figure S1. Mapping calibration of Fluoride. Best fit constants are stored at every $\boldsymbol{\theta}$ time interval. The fluoride peak was as observed on an AS15 column under the stated conditions. 


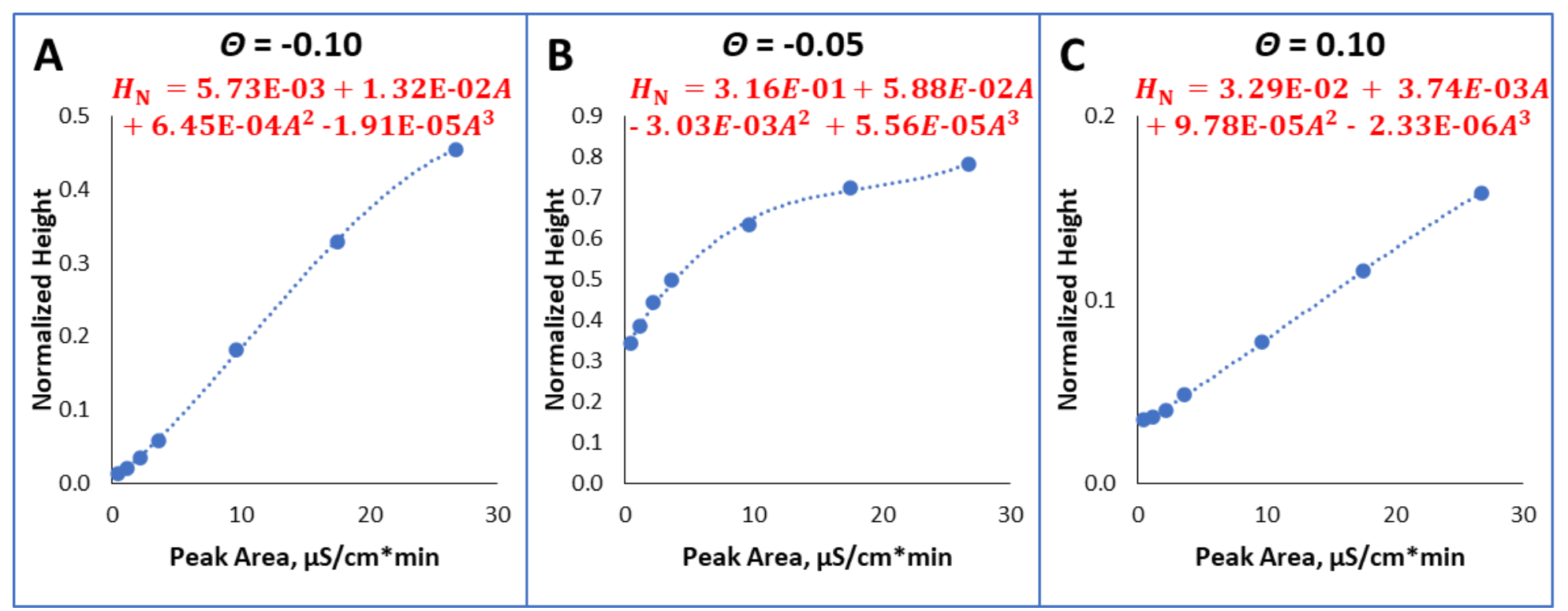

Figure S2. The ability of a third-degree polynomial to predict the fluoride peak shape. Three isolated points are shown in panels A-C for $\theta=-0.10,-.05$ and 0.10 , respectively. The dashed lines depict the best fit equation in red while the solid symbols represent actual experimental data. The raw normalized data set are those shown in Figure S1. 
Table S2. Comparison of Present Strategy (Eq 1) vs. a General Gaussian Model for Identification of Fluoride as Analyte at Different Concentrations

\begin{tabular}{|l|c|c|}
\cline { 2 - 3 } \multicolumn{1}{c|}{} & \multicolumn{2}{c|}{$\boldsymbol{r}^{2}$ for Fluoride identity } \\
\hline Sample & Eq 1 & Gen Gauss \\
\hline $0.100 \mathrm{ppm} \mathrm{F}^{-}$ & 0.9998 & 0.9981 \\
\hline $0.300 \mathrm{ppm} \mathrm{F}^{-}$ & 1.0000 & 0.9984 \\
\hline $0.600 \mathrm{ppm} \mathrm{F}^{-}$ & 1.0000 & 0.9983 \\
\hline $1.00 \mathrm{ppm} \mathrm{F}^{-}$ & 1.0000 & 0.9984 \\
\hline $3.00 \mathrm{ppm} \mathrm{F}^{-}$ & 0.9999 & 0.9974 \\
\hline $6.00 \mathrm{ppm} \mathrm{F}^{-}$ & 0.9999 & 0.9968 \\
\hline $10.0 \mathrm{ppm} \mathrm{F}^{-}$ & 1.0000 & 0.9959 \\
\hline $30.0 \mathrm{ppm} \mathrm{F}$ & 0.9999 & 0.9908 \\
\hline $60.0 \mathrm{ppm} \mathrm{F}^{-}$ & 1.0000 & 0.9793 \\
\hline $100 \mathrm{ppm} \mathrm{F}^{-}$ & 1.0000 & 0.9686 \\
\hline
\end{tabular}



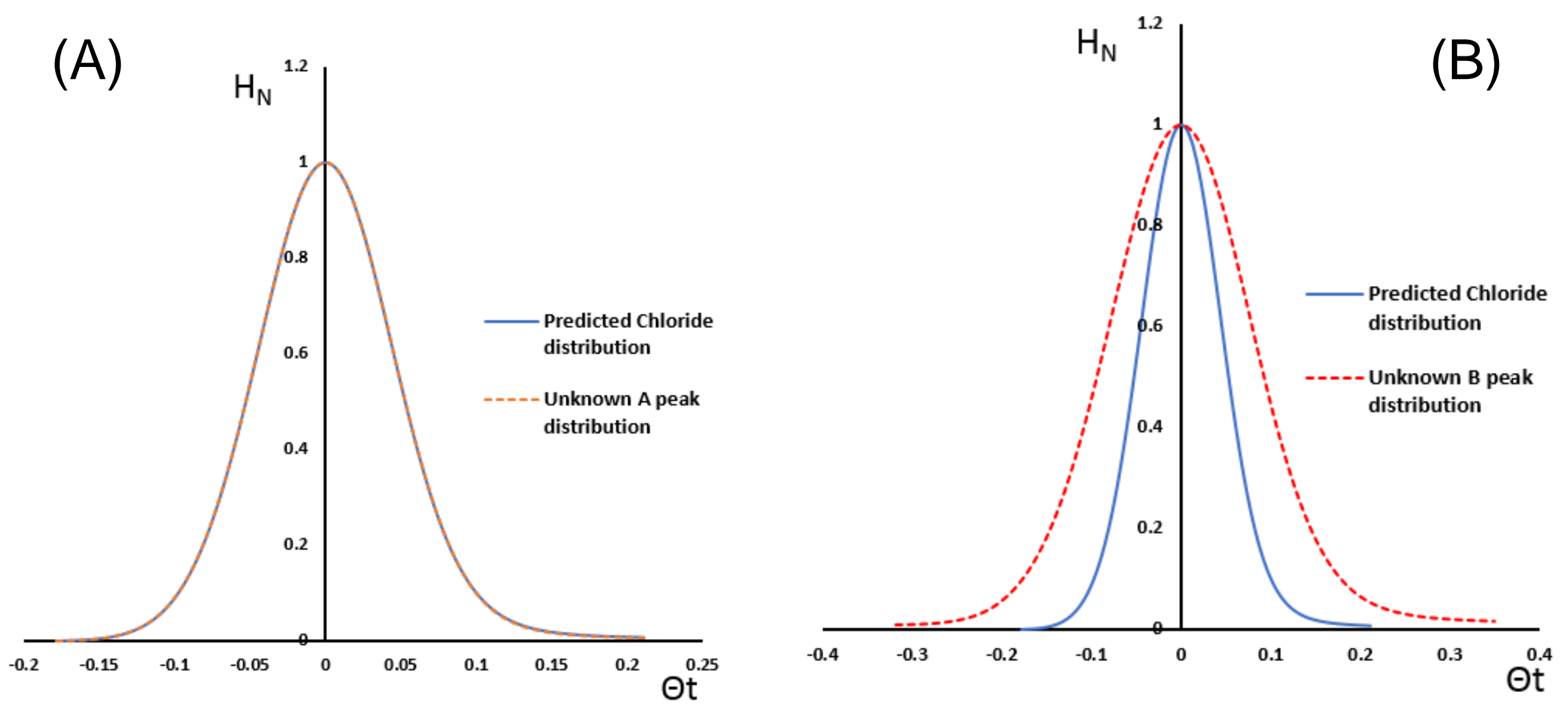

Figure S3. (A) Good match, identity confirmed; (B) Poor match, identity confirmation did not succeed 

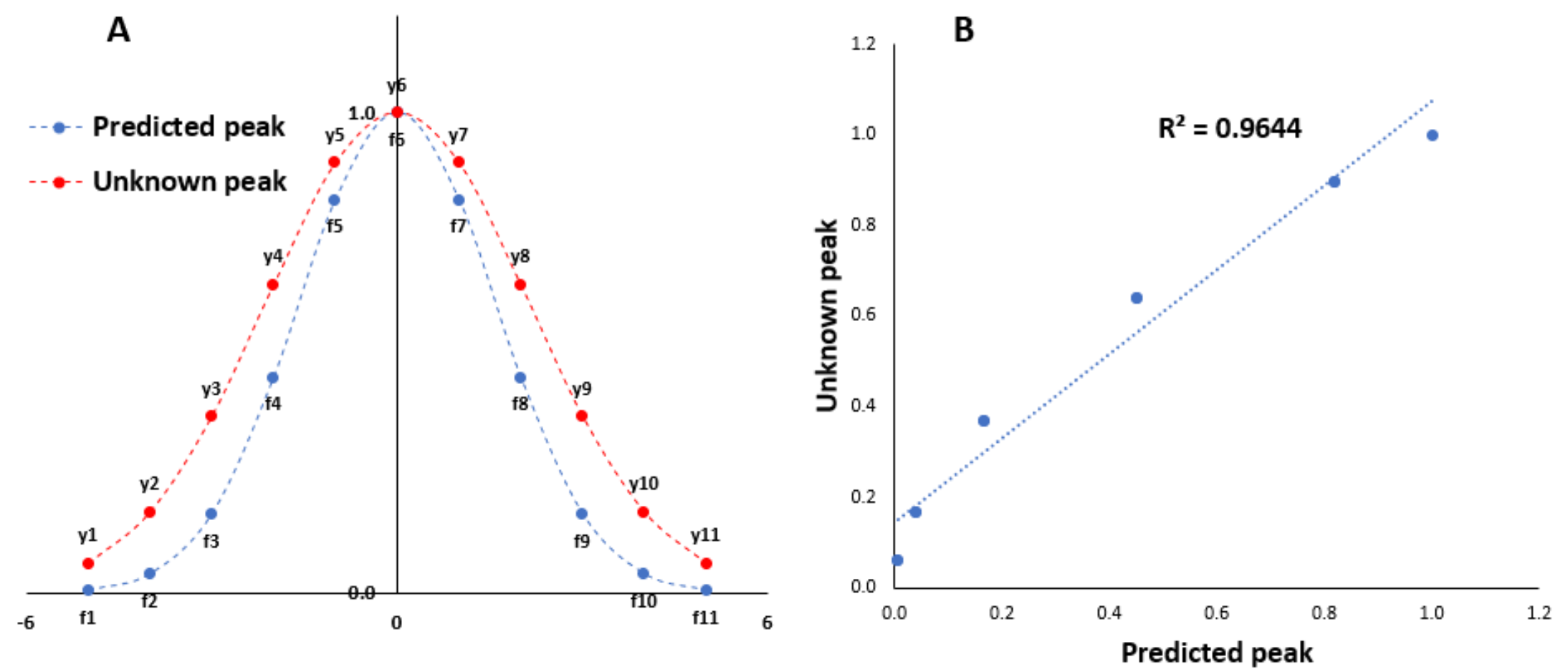

Figure S4. The Coefficient of Determination $r^{2}$, the square of the of the Pearson correlation coefficient $r$ of the Unknown peak data points vs predicted peak data points as an index of goodness of match. The present example, an obvious poor match, exhibits an $r^{2}$ value of 0.9644 . 


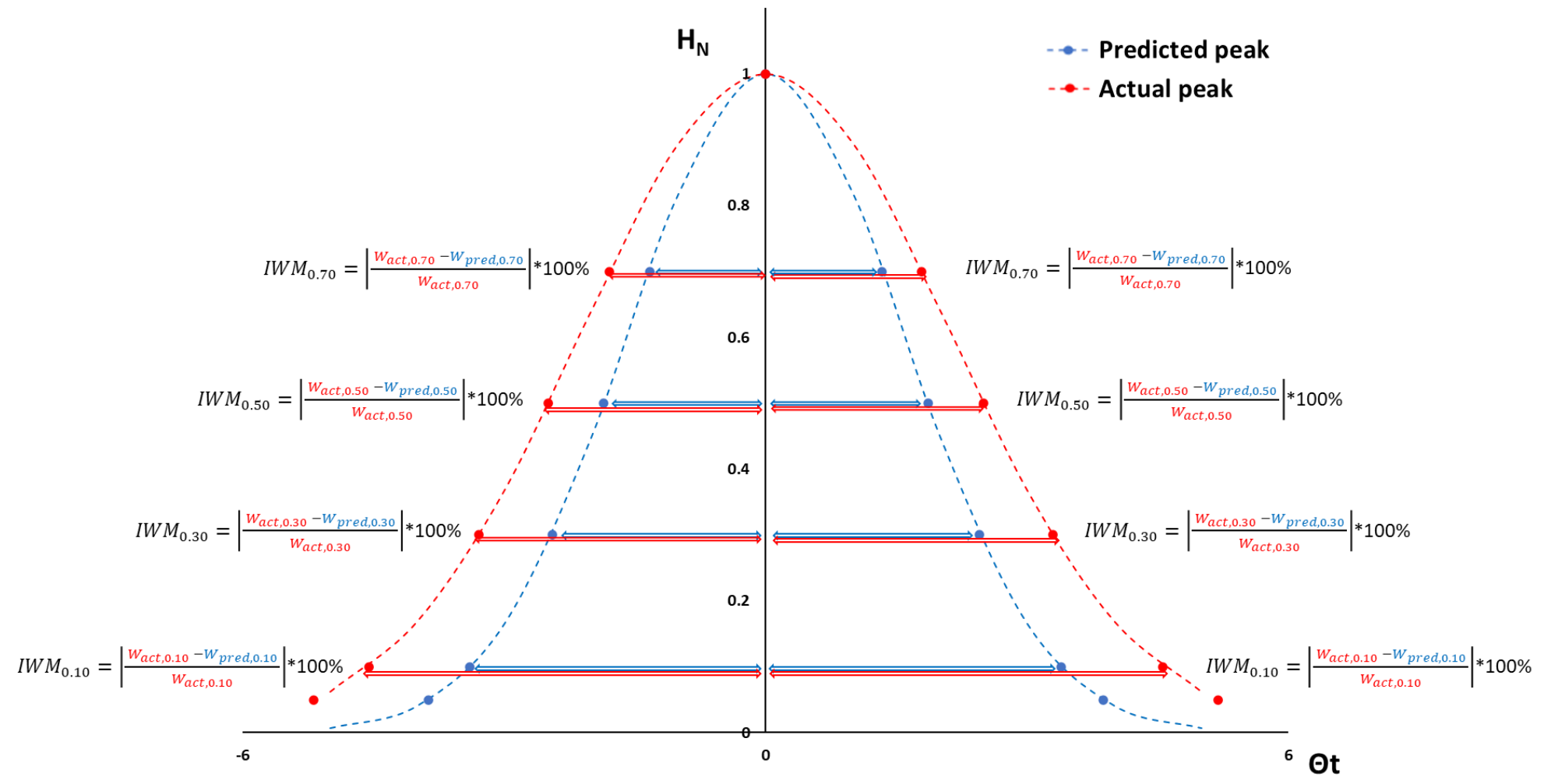

Figure S5. Illustration of the individual Index of Width Mismatch (IWM) computation. IWM is calculated at each individual point in normalized peak height. 


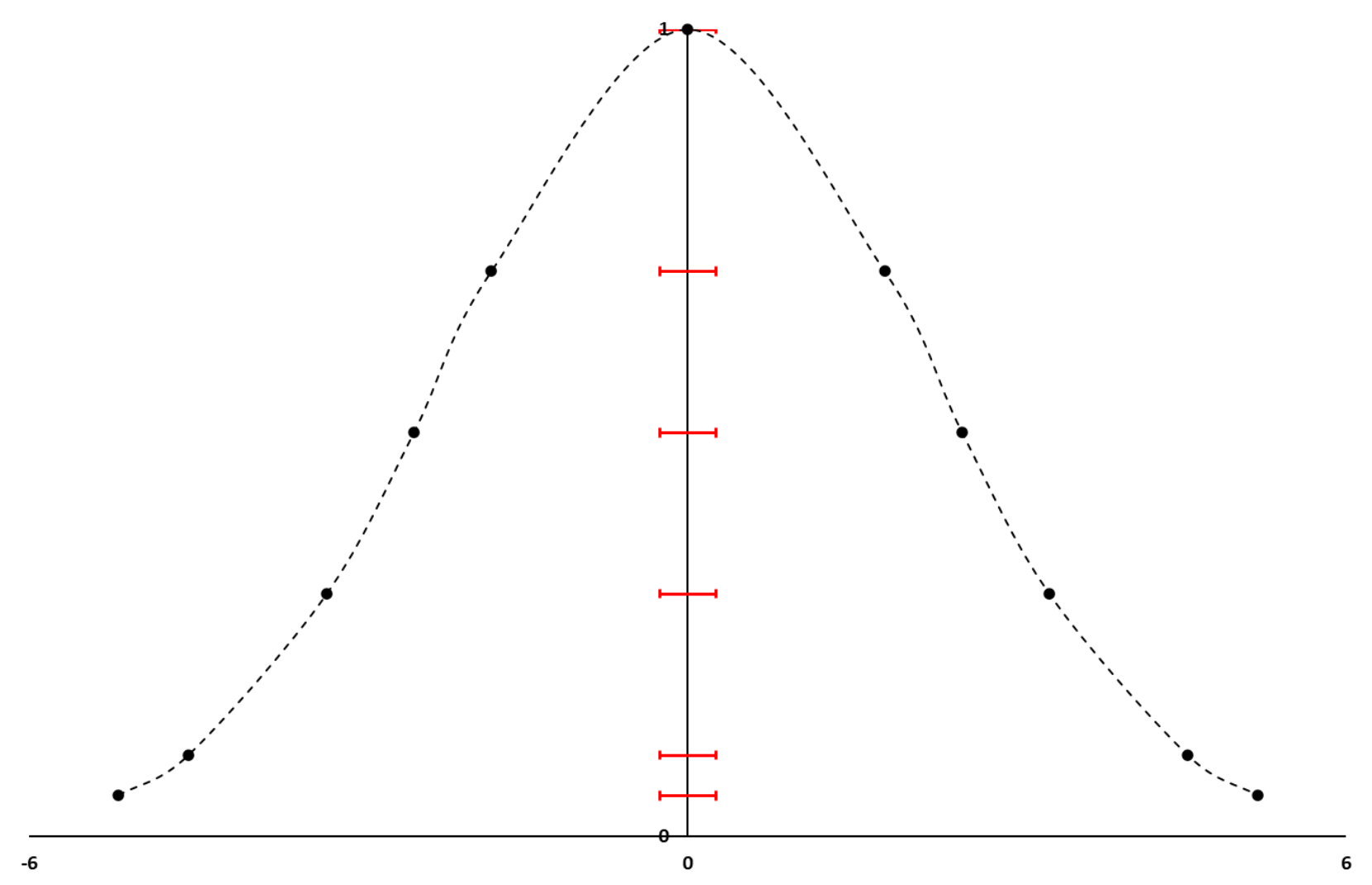

Figure S6. Graphical plot of IWMh for the actual peak in Figure S5. IWMh values are plotted as the $x$-coordinate error bar, centered on $x=0$ in red. 


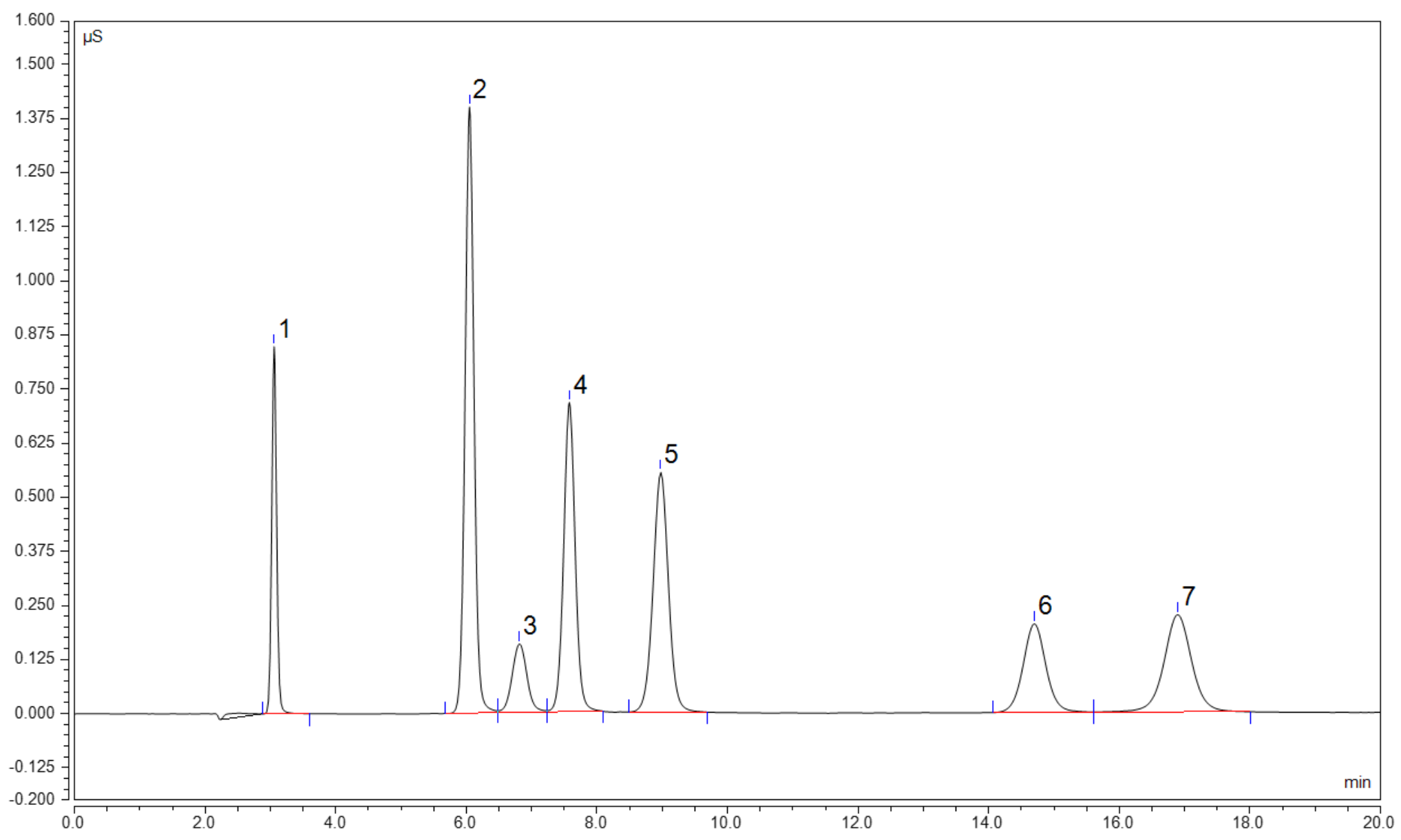

Figure S7. 7 anions standard run on an AS15 column. See main text for experimental conditions. The stock standard (20 ppm $\mathrm{F}$ and $\left.100 \mathrm{ppm} \mathrm{Cl}^{-}, \mathrm{NO}_{2}{ }^{-}, \mathrm{Br}^{-}, \mathrm{NO}_{3}{ }^{-}, \mathrm{SO}_{4}{ }^{2-}\right)$ was injected after various dilutions; the chromatogram shown represents 100x dilution of the stock standard. 
Table S3. AS15 Column Correlation Matrix (unabridged version of Table 4)

\begin{tabular}{|c|c|c|c|c|c|c|}
\hline \multirow[b]{2}{*}{ Sample } & \multicolumn{6}{|c|}{$R^{2}$ values } \\
\hline & Fluoride & Chloride & Nitrite & Sulfate & Bromide & Nitrate \\
\hline $0.020 \mathrm{ppm}$ Fluoride & 1.0000 & 0.8759 & 0.7991 & 0.7289 & 0.6768 & 0.6636 \\
\hline $0.028 \mathrm{ppm}$ Fluoride & 1.0000 & 0.8801 & 0.8079 & 0.7428 & 0.6945 & 0.6832 \\
\hline $0.060 \mathrm{ppm}$ Fluoride & 1.0000 & 0.8759 & 0.8012 & 0.7336 & 0.6838 & 0.6719 \\
\hline $0.200 \mathrm{ppm}$ Fluoride & 1.0000 & 0.8708 & 0.7929 & 0.7227 & 0.6721 & 0.6594 \\
\hline $0.280 \mathrm{ppm}$ Fluoride & 1.0000 & 0.8718 & 0.7924 & 0.7209 & 0.6703 & 0.6572 \\
\hline $0.660 \mathrm{ppm}$ Fluoride & 1.0000 & 0.8809 & 0.8047 & 0.7373 & 0.6923 & 0.6809 \\
\hline $2.00 \mathrm{ppm}$ Fluoride & 1.0000 & 0.8888 & 0.8093 & 0.7428 & 0.7080 & 0.6980 \\
\hline $2.85 \mathrm{ppm}$ Fluoride & 1.0000 & 0.8911 & 0.8101 & 0.7450 & 0.7157 & 0.7068 \\
\hline $6.66 \mathrm{ppm}$ Fluoride & 1.0000 & 0.8990 & 0.8115 & 0.7537 & 0.7376 & 0.7310 \\
\hline $20.0 \mathrm{ppm}$ Fluoride & 1.0000 & 0.9111 & 0.8142 & 0.7672 & 0.7094 & 0.6878 \\
\hline $0.100 \mathrm{ppm}$ Chloride & 0.8394 & 1.0000 & 0.9724 & 0.8864 & 0.7693 & 0.7203 \\
\hline $0.140 \mathrm{ppm}$ Chloride & 0.8419 & 1.0000 & 0.9719 & 0.8878 & 0.7758 & 0.7295 \\
\hline $0.333 \mathrm{ppm}$ Chloride & 0.8479 & 0.9999 & 0.9702 & 0.8898 & 0.7891 & 0.7491 \\
\hline 1.00 ppm Chloride & 0.8454 & 1.0000 & 0.9717 & 0.8940 & 0.7978 & 0.7614 \\
\hline $1.43 \mathrm{ppm}$ Chloride & 0.8458 & 1.0000 & 0.9723 & 0.8964 & 0.8033 & 0.7697 \\
\hline $3.33 \mathrm{ppm}$ Chloride & 0.8503 & 1.0000 & 0.9720 & 0.8984 & 0.8104 & 0.7822 \\
\hline $10.0 \mathrm{ppm}$ Chloride & 0.8694 & 1.0000 & 0.9654 & 0.8972 & 0.8172 & 0.7892 \\
\hline $14.3 \mathrm{ppm}$ Chloride & 0.8771 & 1.0000 & 0.9617 & 0.8964 & 0.8137 & 0.7767 \\
\hline $33.3 \mathrm{ppm}$ Chloride & 0.9096 & 1.0000 & 0.9549 & 0.9025 & 0.7711 & 0.7017 \\
\hline 100 ppm Chloride & 0.5095 & 1.0000 & 0.4837 & 0.7754 & 0.1035 & 0.0436 \\
\hline 0.100 ppm Nitrite & 0.7139 & 0.9692 & 0.9999 & 0.9711 & 0.8962 & 0.8607 \\
\hline 0.140 ppm Nitrite & 0.7169 & 0.9704 & 1.0000 & 0.9692 & 0.8908 & 0.8533 \\
\hline 0.333 ppm Nitrite & 0.7199 & 0.9714 & 1.0000 & 0.9643 & 0.8720 & 0.8272 \\
\hline 1.00 ppm Nitrite & 0.7209 & 0.9715 & 1.0000 & 0.9607 & 0.8561 & 0.8055 \\
\hline $1.43 \mathrm{ppm}$ Nitrite & 0.7195 & 0.9703 & 1.0000 & 0.9615 & 0.8573 & 0.8082 \\
\hline $3.33 \mathrm{ppm}$ Nitrite & 0.7170 & 0.9666 & 1.0000 & 0.9650 & 0.8648 & 0.8206 \\
\hline $10.0 \mathrm{ppm}$ Nitrite & 0.7239 & 0.9626 & 1.0000 & 0.9685 & 0.8738 & 0.8351 \\
\hline $14.3 \mathrm{ppm}$ Nitrite & 0.7275 & 0.9613 & 1.0000 & 0.9696 & 0.8751 & 0.8331 \\
\hline $33.3 \mathrm{ppm}$ Nitrite & 0.7347 & 0.9536 & 1.0000 & 0.9751 & 0.8721 & 0.8079 \\
\hline 100 ppm Nitrite & 0.6107 & 0.9036 & 1.0000 & 0.9900 & 0.7866 & 0.6711 \\
\hline $0.100 \mathrm{ppm}$ Sulfate & 0.5576 & 0.8610 & 0.9540 & 0.9998 & 0.9533 & 0.9127 \\
\hline $0.140 \mathrm{ppm}$ Sulfate & 0.5629 & 0.8656 & 0.9570 & 0.9999 & 0.9552 & 0.9164 \\
\hline $0.330 \mathrm{ppm}$ Sulfate & 0.5698 & 0.8706 & 0.9600 & 1.0000 & 0.9474 & 0.9031 \\
\hline $1.00 \mathrm{ppm}$ Sulfate & 0.5745 & 0.8736 & 0.9625 & 0.9999 & 0.9414 & 0.8935 \\
\hline $1.43 \mathrm{ppm}$ Sulfate & 0.5734 & 0.8715 & 0.9618 & 0.9999 & 0.9428 & 0.8962 \\
\hline $3.33 \mathrm{ppm}$ Sulfate & 0.5716 & 0.8651 & 0.9595 & 1.0000 & 0.9450 & 0.8991 \\
\hline $10.0 \mathrm{ppm}$ Sulfate & 0.5877 & 0.8655 & 0.9642 & 1.0000 & 0.9412 & 0.8908 \\
\hline $14.3 \mathrm{ppm}$ Sulfate & 0.5959 & 0.8666 & 0.9669 & 1.0000 & 0.9368 & 0.8794 \\
\hline $33.3 \mathrm{ppm}$ Sulfate & 0.6238 & 0.8688 & 0.9736 & 1.0000 & 0.9093 & 0.8295 \\
\hline 100 ppm Sulfate & 0.5073 & 0.8401 & 0.9935 & 1.0000 & 0.4875 & 0.5062 \\
\hline 0.100 ppm Bromide & 0.4031 & 0.6884 & 0.8118 & 0.9380 & 0.9993 & 0.9898 \\
\hline $0.140 \mathrm{ppm}$ Bromide & 0.3862 & 0.6667 & 0.7920 & 0.9256 & 0.9990 & 0.9900 \\
\hline 0.333 ppm Bromide & 0.3967 & 0.6780 & 0.8019 & 0.9324 & 0.9999 & 0.9892 \\
\hline $1.00 \mathrm{ppm}$ Bromide & 0.4039 & 0.6853 & 0.8090 & 0.9366 & 0.9999 & 0.9851 \\
\hline $1.43 \mathrm{ppm}$ Bromide & 0.4047 & 0.6853 & 0.8097 & 0.9369 & 0.9999 & 0.9848 \\
\hline 3.33 ppm Bromide & 0.4083 & 0.6865 & 0.8129 & 0.9376 & 0.9999 & 0.9840 \\
\hline 10.0 ppm Bromide & 0.4125 & 0.6801 & 0.8137 & 0.9323 & 1.0000 & 0.9864 \\
\hline $14.3 \mathrm{ppm}$ Bromide & 0.4167 & 0.6792 & 0.8160 & 0.9301 & 1.0000 & 0.9865 \\
\hline $33.3 \mathrm{ppm}$ Bromide & 0.4294 & 0.6754 & 0.8216 & 0.9202 & 1.0000 & 0.9822 \\
\hline 100 ppm Bromide & 0.4637 & 0.6319 & 0.7907 & 0.8700 & 1.0000 & 0.9780 \\
\hline 0.100 ppm Nitrate & 0.3249 & 0.5781 & 0.7019 & 0.8555 & 0.9828 & 0.9993 \\
\hline $0.140 \mathrm{ppm}$ Nitrate & 0.3263 & 0.5790 & 0.7021 & 0.8556 & 0.9835 & 0.9998 \\
\hline $0.333 \mathrm{ppm}$ Nitrate & 0.3363 & 0.5932 & 0.7172 & 0.8678 & 0.9869 & 0.9999 \\
\hline $1.00 \mathrm{ppm}$ Nitrate & 0.3422 & 0.5964 & 0.7194 & 0.8676 & 0.9877 & 0.9999 \\
\hline $1.43 \mathrm{ppm}$ Nitrate & 0.3435 & 0.5974 & 0.7216 & 0.8689 & 0.9879 & 0.9997 \\
\hline $3.33 \mathrm{ppm}$ Nitrate & 0.3462 & 0.5982 & 0.7259 & 0.8703 & 0.9883 & 0.9997 \\
\hline $10.0 \mathrm{ppm}$ Nitrate & 0.3512 & 0.5906 & 0.7269 & 0.8601 & 0.9840 & 1.0000 \\
\hline $14.3 \mathrm{ppm}$ Nitrate & 0.3540 & 0.5879 & 0.7281 & 0.8553 & 0.9828 & 0.9999 \\
\hline $33.3 \mathrm{ppm}$ Nitrate & 0.3581 & 0.5716 & 0.7196 & 0.8279 & 0.9795 & 1.0000 \\
\hline 100 ppm Nitrate & 0.3679 & 0.4986 & 0.6663 & 0.7334 & 0.9682 & 1.0000 \\
\hline
\end{tabular}




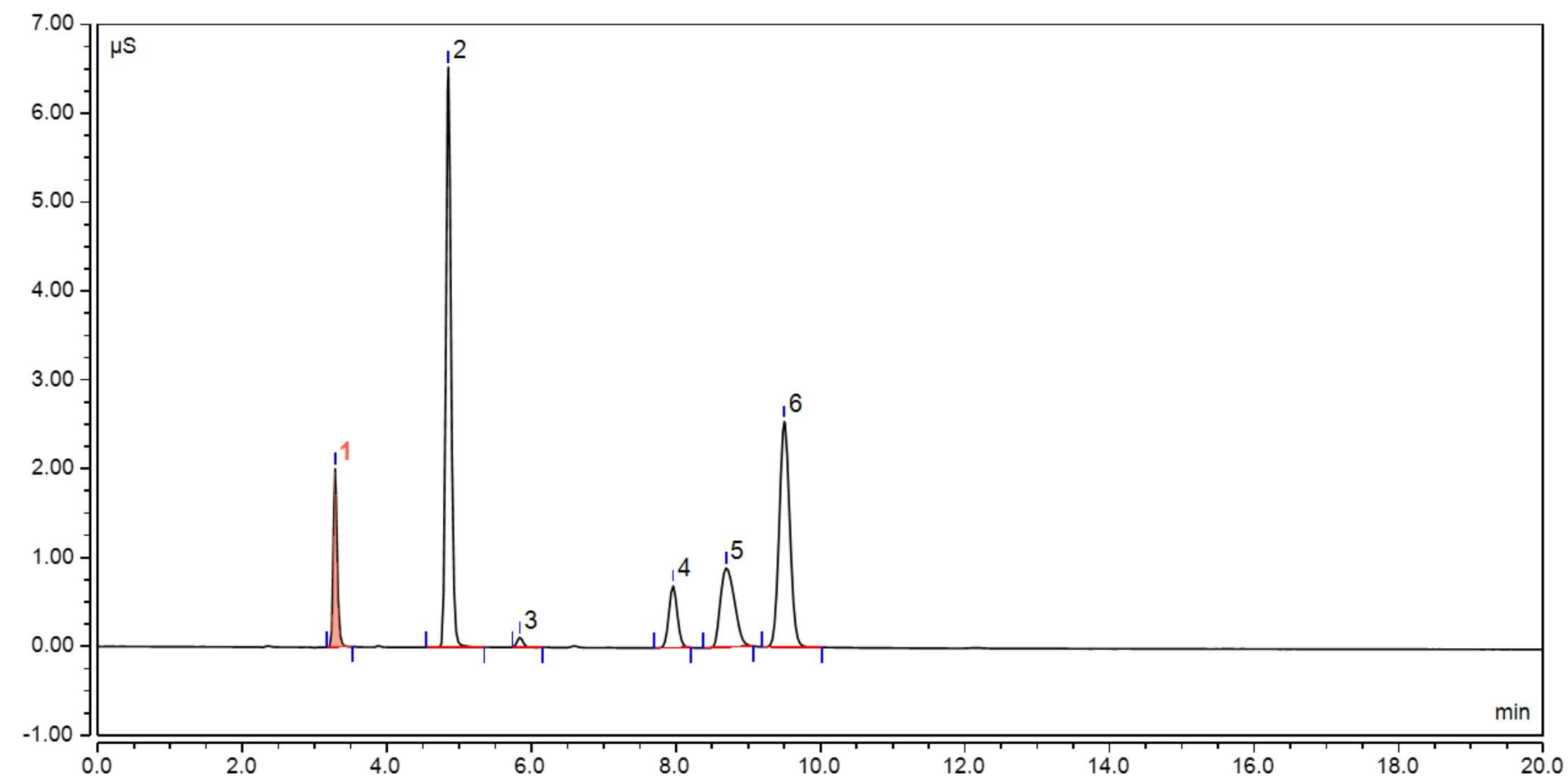

Figure S8. Chromatogram of a drinking water sample separated on an AS19 $4 \times 150 \mathrm{~mm} 4 \mu \mathrm{m}$ column. Conditions given in main text. 


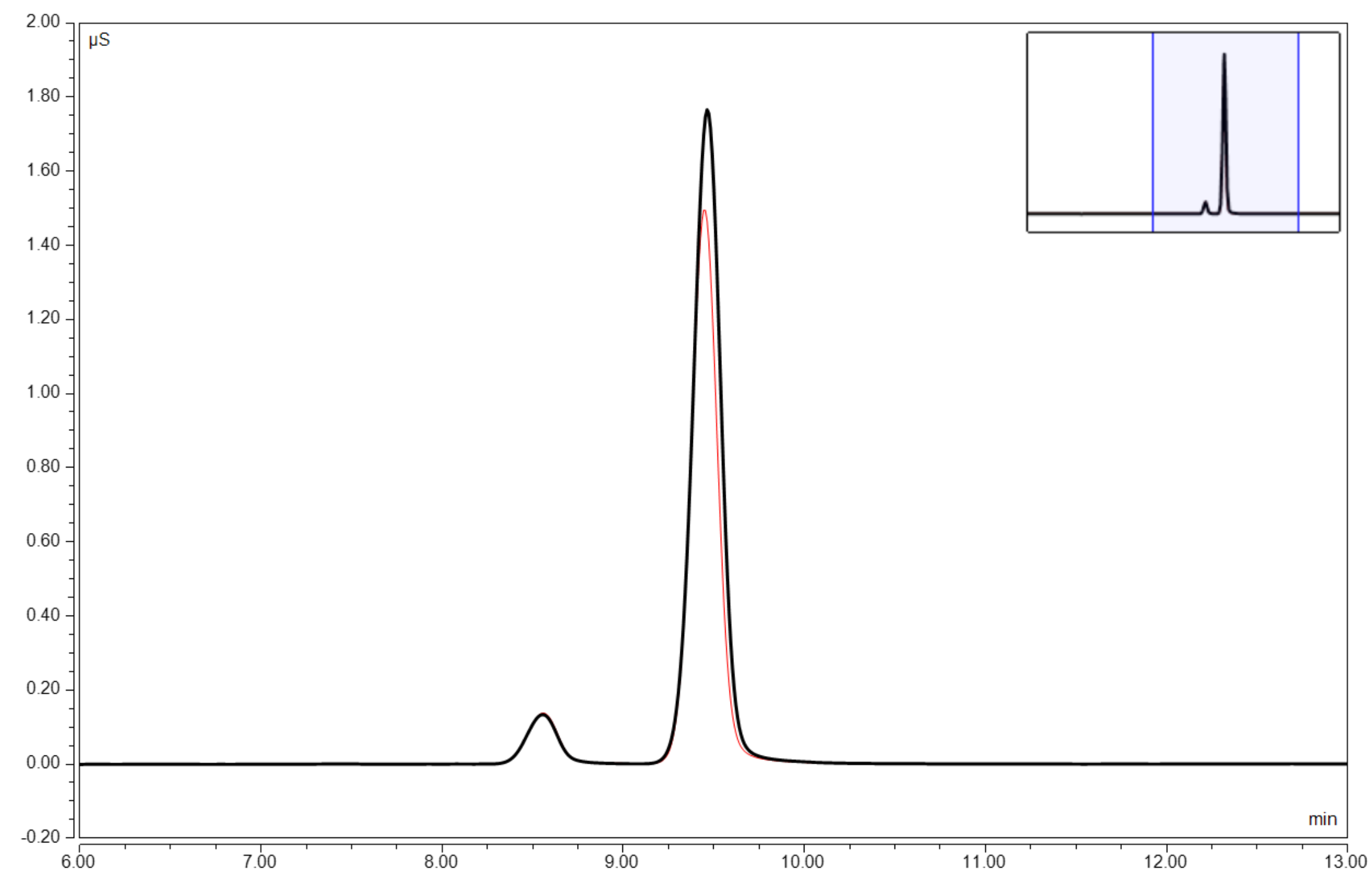

Figure S9. Overlaid chromatograms of 3 ppm Sulfate (black) and 3 ppm Nitrate (red) on an lon Pac AS19 2x250 mm column with 25.5 $\mathrm{mM} \mathrm{KOH}$ eluent. Conditions given in main text. The first peak is carbonate, not deliberately added. 


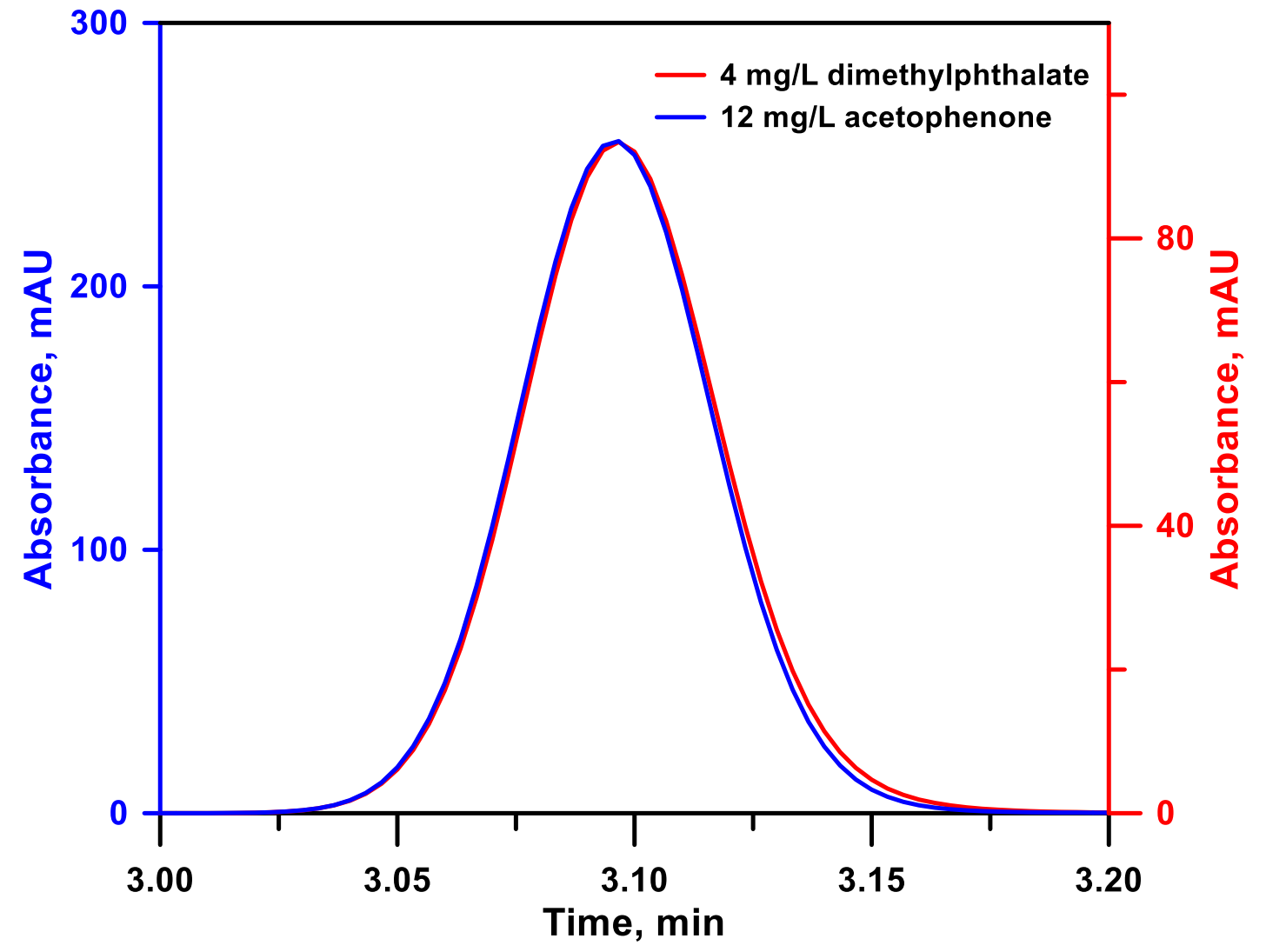

Figure S10. Overlaid chromatograms of 4 ppm dimethyl phthalate (red) and 12 ppm acetophenone (blue). The HPLC equipment, including the column was the same as that described in the Experimental section. An isocratic eluent containing acetonitrile and 100 $\mathrm{mM} \mathrm{NH}_{4} \mathrm{OAc}(\mathrm{pH} 5)$ aqueous buffer (57:43 volume ratio) was used. The numerical data has a coefficient of determination $\left(r^{2}\right)$ of 0.9982 when the normalized versions are compared against each other.. 
Table S4. Identity Correlation Matrix, Anion Separation on AS 18 Column $R^{2}$ values

\begin{tabular}{|c|c|c|c|c|c|c|}
\hline Sample & Fluoride & Chloride & Nitrite & Bromide & Sulfate & Nitrate \\
\hline $0.020 \mathrm{ppm}$ Fluoride & 0.9997 & 0.9873 & 0.9592 & 0.8648 & 0.8180 & 0.7832 \\
\hline $0.028 \mathrm{ppm}$ Fluoride & .9999 & 0.9893 & 0.9632 & 0.8769 & 0.8353 & 0.8071 \\
\hline 0.060 ppm Fluoride & 9999 & 0.9904 & 0.9650 & 0.8815 & 0.8414 & 0.8152 \\
\hline $0.200 \mathrm{ppm}$ Fluoride & 1.0000 & 0.9920 & 0.9675 & 0.8885 & 0.8501 & 0.8223 \\
\hline $0.280 \mathrm{ppm}$ Fluoride & 0.9999 & 0.9923 & 0.9677 & 0.8900 & 0.8516 & 0.8222 \\
\hline $0.660 \mathrm{ppm}$ Fluoride & 0.9999 & 0.9943 & 0.9700 & 0.8965 & 0.8568 & 0.8232 \\
\hline 2.00 ppm Fluoride & 1.0000 & 0.9968 & 0.9701 & 0.8973 & 0.8580 & 0.8077 \\
\hline $2.85 \mathrm{ppm}$ Fluoride & 0.9999 & 0.9974 & 0.9686 & 0.8947 & 0.8548 & 0.7916 \\
\hline $6.66 \mathrm{ppm}$ Fluoride & 1.0000 & 0.9979 & 0.9613 & 0.8738 & 0.8266 & 0.7107 \\
\hline 20.0 ppm Fluoride & 1.0000 & 0.9906 & 0.9447 & 0.0159 & 0.3700 & 0.6508 \\
\hline 0.100 ppm Chloride & 0.9908 & 1.0000 & 0.9912 & 0.9342 & 0.9026 & 0.8797 \\
\hline 0.140 ppm Chloride & 0.9912 & 1.0000 & 0.9910 & 0.9334 & 0.9011 & 0.8781 \\
\hline 0.333 ppm Chloride & 0.9916 & 1.0000 & 0.9908 & 0.9337 & 0.9009 & 0.8768 \\
\hline $1.00 \mathrm{ppm}$ Chloride & 0.9924 & 1.0000 & 0.9907 & 0.9356 & 0.9010 & 0.8744 \\
\hline $1.43 \mathrm{ppm}$ Chloride & 0.9932 & 1.0000 & 0.9902 & 0.9353 & 0.9001 & 0.8710 \\
\hline 3.33 ppm Chloride & 0.9958 & 0.9999 & 0.9875 & 0.9297 & 0.8948 & 0.8503 \\
\hline 10.0 ppm Chloride & 0.9979 & 1.0000 & 0.9738 & 0.9284 & 0.8596 & 0.7571 \\
\hline $14.3 \mathrm{ppm}$ Chlo & 0.9969 & 1.0000 & 0.9643 & 0.4132 & 0.8410 & 0.7084 \\
\hline $33.3 \mathrm{ppm}$ Chloride & 0.9874 & 1.0000 & 0.9310 & 0.0055 & 0.1449 & 0.6733 \\
\hline 100 ppm Chlor & 0.2608 & 1.0000 & 0.4859 & 0.0006 & 0.0071 & 0.0049 \\
\hline 0.100 & 0.9657 & 0.9919 & 1.0000 & 0.9699 & 0.9446 & 0.9261 \\
\hline 0.140 & 0.9661 & 0.9921 & 1.0000 & 0.9696 & 0.9444 & 0.9247 \\
\hline 0.333 ppm Nitrite & 0.9664 & 0.9918 & 1.0000 & 0.9699 & 0.9436 & 0.9242 \\
\hline $1.00 \mathrm{ppm}$ Nitrite & 0.9664 & 0.9904 & 0.9999 & 0.9717 & 0.9448 & 0.9236 \\
\hline 1.43 ppm Nitrite & 0.9664 & 0.9895 & 1.0000 & 0.9730 & 0.9457 & 0.9239 \\
\hline 3.33 ppm Nitrite & 0.9675 & 0.9865 & 1.0000 & 0.9746 & 0.9480 & 0.9190 \\
\hline & .9679 & 0.9785 & 1.0000 & .9798 & 0.9436 & 0.8803 \\
\hline 14.3 ppm Nitrite & 0.9646 & 0.9736 & 1.0000 & 0.9693 & 0.9362 & 0.8520 \\
\hline 33.3 ppm Nitrite & 0.9367 & 0.9475 & 1.0000 & 0.0107 & 0.9431 & 0.7990 \\
\hline 100 ppm Nitrite & 0.7225 & 0.8377 & 1.0000 & 0.0176 & 0.0199 & 0.6100 \\
\hline 0.100 ppm Bromide & 0.8766 & 0.9295 & 0.9677 & 1.0000 & 0.9953 & 0.9880 \\
\hline 0.140 ppm Bromide & 0.8787 & 0.9308 & 0.9687 & 1.0000 & 0.9947 & 0.9871 \\
\hline 0.333 ppm Bromide & 0.8793 & 0.9307 & 0.9688 & 1.0000 & 0.9949 & 0.9867 \\
\hline ppm Bromide & 0.8835 & 0.9322 & 0.9707 & 0.9999 & 0.9943 & 0.9849 \\
\hline 1.43 ppm Bromide & 0.8847 & 0.9318 & 0.9709 & 1.0000 & 0.9943 & 0.9845 \\
\hline 3.33 ppm Bromide & 0.8882 & 0.9291 & 0.9713 & 1.0000 & 0.9944 & 0.9836 \\
\hline 10.0 ppm Bromide & 0.8959 & 0.9222 & 0.9717 & 1.0000 & 0.9939 & 0.9746 \\
\hline 14.3 ppm Bromide & 0.8987 & 0.9201 & 0.9731 & 1.0000 & 0.9936 & 0.9647 \\
\hline 0.100 ppm Sulfate & 0.8313 & 0.8893 & 0.9370 & 0.9942 & 1.0000 & 0.9978 \\
\hline 0.140 ppm Sulfate & 0.8321 & 0.8900 & 0.9378 & 0.9943 & 1.0000 & 0.9976 \\
\hline $0.330 \mathrm{ppm}$ Sulfate & 0.8353 & 0.8911 & 0.9391 & 0.9945 & 1.0000 & 0.9968 \\
\hline 1.00 ppm Sulfate & 0.8385 & 0.8898 & 0.9400 & 0.9942 & 1.0000 & 0.9960 \\
\hline 1.43 ppm Sulfate & 0.8388 & 0.8877 & 0.9396 & 0.9937 & 1.0000 & 0.9958 \\
\hline 3.33 ppm Sulfate & 0.8448 & 0.8835 & 0.9415 & 0.9932 & 1.0000 & 0.9931 \\
\hline $10.0 \mathrm{ppm}$ Sulfate & 0.8463 & 0.8678 & 0.9417 & 0.9890 & 1.0000 & 0.9820 \\
\hline 14.3 ppm Sulfate & 0.8349 & 0.8548 & 0.9370 & 0.8773 & 1.0000 & 0.9766 \\
\hline 0.100 ppm Nitrate & 0.8026 & 0.8634 & 0.9154 & 0.9859 & 0.9977 & 0.9999 \\
\hline 0.140 ppm Nitrate & 0.8013 & 0.8613 & 0.9136 & 0.9849 & 0.9971 & 1.0000 \\
\hline $0.333 \mathrm{ppm}$ Nitrate & 0.8042 & 0.8627 & 0.9152 & 0.9854 & 0.9977 & 1.0000 \\
\hline 1.00 ppm Nitrate & 0.8056 & 0.8603 & 0.9146 & 0.9839 & 0.9968 & 1.0000 \\
\hline 1.43 ppm Nitrate & 0.8051 & 0.8579 & 0.9136 & 0.9826 & 0.9960 & 1.0000 \\
\hline 3.33 ppm Nitrate & 0.8043 & 0.8488 & 0.9099 & 0.9785 & 0.9935 & 0.9999 \\
\hline 10.0 ppm Nitrate & 0.7917 & 0.8182 & 0.8942 & 0.9679 & 0.9869 & 1.0000 \\
\hline $14.3 \mathrm{ppm}$ Nitrate & 0.7743 & 0.7964 & 0.8798 & 0.9373 & 0.9829 & 1.0000 \\
\hline $33.3 \mathrm{ppm}$ Nitrate & 0.6942 & 0.7198 & 0.8305 & 0.0003 & 0.9457 & 1.0000 \\
\hline 100 ppm Nitrate & 0.6378 & 0.5565 & 0.7383 & 0.1613 & 0.0058 & 1.0000 \\
\hline
\end{tabular}


Table S5. Identity Correlation Matrix, Anion Separation on AS 19 Column

\begin{tabular}{|c|c|c|c|c|c|c|}
\hline & \multicolumn{6}{|c|}{$\mathbf{R}^{2}$ values } \\
\hline Sample & Fluoride & Chloride & Nitrite & Bromide & Sulfate & Nitrate \\
\hline 0.100 ppm Fluoride & 0.9998 & 0.9621 & 0.9066 & 0.8491 & 0.8095 & 0.7500 \\
\hline 0.300 ppm Fluoride & 1.0000 & 0.9642 & 0.9062 & 0.8491 & 0.8090 & 0.7477 \\
\hline 0.600 ppm Fluoride & 1.0000 & 0.9669 & 0.9073 & 0.8541 & 0.8155 & 0.7558 \\
\hline 1.00 ppm Fluoride & 1.0000 & 0.9703 & 0.9094 & 0.8622 & 0.8264 & 0.7703 \\
\hline $3.00 \mathrm{ppm}$ Fluoride & 0.9999 & 0.9796 & 0.9123 & 0.8857 & 0.8561 & 0.8129 \\
\hline $6.00 \mathrm{ppm}$ Fluoride & 0.9999 & 0.9865 & 0.9122 & 0.8955 & 0.8587 & 0.8161 \\
\hline $10.0 \mathrm{ppm}$ Fluoride & 1.0000 & 0.9895 & 0.8978 & 0.8773 & 0.8245 & 0.7675 \\
\hline 30.0 ppm Fluoride & 0.9999 & 0.9818 & 0.8988 & 0.7716 & 0.6485 & 0.5276 \\
\hline 60.0 ppm Fluoride & 1.0000 & 0.9445 & 0.8839 & 0.8631 & 0.5156 & 0.3614 \\
\hline 100.0 ppm Fluoride & 1.0000 & 0.8937 & 0.7524 & 0.2774 & 0.5516 & 0.3412 \\
\hline 0.100 ppm Chloride & 0.9593 & 1.0000 & 0.9814 & 0.9336 & 0.8911 & 0.8114 \\
\hline 0.300 ppm Chloride & 0.9608 & 1.0000 & 0.9792 & 0.9279 & 0.8815 & 0.7933 \\
\hline 0.600 ppm Chloride & 0.9615 & 1.0000 & 0.9791 & 0.9305 & 0.8867 & 0.8046 \\
\hline 1.00 ppm Chloride & 0.9626 & 1.0000 & 0.9783 & 0.9315 & 0.8886 & 0.8095 \\
\hline 3.00 ppm Chloride & 0.9702 & 1.0000 & 0.9728 & 0.9346 & 0.8952 & 0.8294 \\
\hline 6.00 ppm Chloride & 0.9793 & 1.0000 & 0.9634 & 0.9359 & 0.8962 & 0.8397 \\
\hline 10.0 ppm Chloride & 0.9866 & 1.0000 & 0.9500 & 0.9324 & 0.8855 & 0.8250 \\
\hline 30.0 ppm Chloride & 0.9828 & 1.0000 & 0.9032 & 0.8474 & 0.7372 & 0.6183 \\
\hline 60.0 ppm Chloride & 0.9413 & 1.0000 & 0.8332 & 0.9097 & 0.5857 & 0.4640 \\
\hline 100 ppm Chloride & 0.8699 & 1.0000 & 0.2147 & 0.5429 & 0.6584 & 0.2144 \\
\hline 0.100 ppm Nitrite & 0.8867 & 0.9797 & 0.9998 & 0.9820 & 0.9538 & 0.8879 \\
\hline $0.300 \mathrm{ppm}$ Nitrite & 0.8869 & 0.9797 & 1.0000 & 0.9794 & 0.9468 & 0.8655 \\
\hline 0.600 ppm Nitrite & 0.8865 & 0.9790 & 1.0000 & 0.9788 & 0.9445 & 0.8574 \\
\hline 1.00 ppm Nitrite & 0.8863 & 0.9781 & 1.0000 & 0.9808 & 0.9493 & 0.8726 \\
\hline 3.00 ppm Nitrite & 0.8835 & 0.9724 & 1.0000 & 0.9827 & 0.9490 & 0.8645 \\
\hline 6.00 ppm Nitrite & 0.8838 & 0.9655 & 0.9999 & 0.9843 & 0.9502 & 0.8697 \\
\hline $10.0 \mathrm{ppm}$ Nitrite & 0.8867 & 0.9585 & 1.0000 & 0.9844 & 0.9482 & 0.8681 \\
\hline 30.0 ppm Nitrite & 0.8971 & 0.9270 & 1.0000 & 0.9592 & 0.9082 & 0.8163 \\
\hline 600 ppm Nitrite & 0.9016 & 0.8878 & 1.0000 & 0.9080 & 0.8267 & 0.7148 \\
\hline 100 ppm Nitrite & 0.8988 & 0.8403 & 1.0000 & 0.8940 & 0.7555 & 0.6160 \\
\hline 0.100 ppm Bromide & 0.7761 & 0.9104 & 0.9737 & 0.9996 & 0.9926 & 0.9419 \\
\hline 0.300 ppm Bromide & 0.7854 & 0.9171 & 0.9778 & 1.0000 & 0.9912 & 0.9395 \\
\hline 0.600 ppm Bromide & 0.7875 & 0.9178 & 0.9787 & 1.0000 & 0.9899 & 0.9314 \\
\hline 1.00 ppm Bromide & 0.7901 & 0.9188 & 0.9797 & 0.9999 & 0.9891 & 0.9282 \\
\hline 3.00 ppm Bromide & 0.7956 & 0.9187 & 0.9814 & 0.9999 & 0.9891 & 0.9300 \\
\hline $6.00 \mathrm{ppm}$ Bromide & 0.8048 & 0.9189 & 0.9835 & 1.0000 & 0.9892 & 0.9343 \\
\hline $10.0 \mathrm{ppm}$ Bromide & 0.8152 & 0.9186 & 0.9845 & 1.0000 & 0.9890 & 0.9353 \\
\hline 30.0 ppm Bromide & 0.8484 & 0.9121 & 0.9731 & 1.0000 & 0.9861 & 0.9312 \\
\hline 60.0 ppm Bromide & 0.8421 & 0.8820 & 0.9311 & 1.0000 & 0.9799 & 0.9134 \\
\hline 100 ppm Bromide & 0.7874 & 0.8303 & 0.8787 & 1.0000 & 0.9695 & 0.8953 \\
\hline 0.100 ppm Nitrate & 0.7059 & 0.8529 & 0.9361 & 0.9893 & 0.9999 & 0.9734 \\
\hline $0.300 \mathrm{ppm}$ Nitrate & 0.7090 & 0.8546 & 0.9377 & 0.9899 & 1.0000 & 0.9697 \\
\hline $0.600 \mathrm{ppm}$ Nitrate & 0.7118 & 0.8558 & 0.9392 & 0.9902 & 1.0000 & 0.9694 \\
\hline 1.00 ppm Nitrate & 0.7138 & 0.8560 & 0.9402 & 0.9903 & 1.0000 & 0.9675 \\
\hline 3.00 ppm Nitrate & 0.7209 & 0.8551 & 0.9433 & 0.9896 & 1.0000 & 0.9689 \\
\hline 6.00 ppm Nitrate & 0.7326 & 0.8544 & 0.9468 & 0.9887 & 1.0000 & 0.9694 \\
\hline 10.0 ppm Nitrate & 0.7450 & 0.8523 & 0.9484 & 0.9873 & 1.0000 & 0.9707 \\
\hline 30.0 ppm Nitrate & 0.7653 & 0.8210 & 0.9148 & 0.9801 & 1.0000 & 0.9692 \\
\hline 60.0 ppm Nitrate & 0.7189 & 0.7483 & 0.8374 & 0.9730 & 1.0000 & 0.9659 \\
\hline 100 ppm Nitrate & 0.6292 & 0.6650 & 0.7560 & 0.9386 & 1.0000 & 0.9586 \\
\hline $0.100 \mathrm{ppm}$ Sulfate & 0.5704 & 0.7203 & 0.8246 & 0.9200 & 0.9655 & 0.9999 \\
\hline $0.300 \mathrm{ppm}$ Sulfate & 0.5722 & 0.7209 & 0.8254 & 0.9196 & 0.9654 & 1.0000 \\
\hline $0.600 \mathrm{ppm}$ Sulfate & 0.5751 & 0.7226 & 0.8279 & 0.9204 & 0.9660 & 1.0000 \\
\hline 1.00 ppm Sulfate & 0.5774 & 0.7230 & 0.8299 & 0.9205 & 0.9662 & 1.0000 \\
\hline 3.00 ppm Sulfate & 0.5860 & 0.7223 & 0.8359 & 0.9185 & 0.9660 & 1.0000 \\
\hline 6.00 ppm Sulfate & 0.5985 & 0.7210 & 0.8431 & 0.9156 & 0.9657 & 0.9999 \\
\hline 10.0 ppm Sulfate & 0.6126 & 0.7183 & 0.8480 & 0.9118 & 0.9653 & 1.0000 \\
\hline 30.0 ppm Sulfate & 0.6238 & 0.6669 & 0.8052 & 0.8842 & 0.9556 & 0.9999 \\
\hline 60.0 ppm Sulfate & 0.5730 & 0.5780 & 0.7128 & 0.8500 & 0.9468 & 1.0000 \\
\hline 100 ppm Sulfate & 0.4997 & 0.4996 & 0.6317 & 0.7742 & 0.9394 & 1.0000 \\
\hline
\end{tabular}


Table S6. Identity Correlation Matrix, Anion Separation on AS 22 Column

\begin{tabular}{|c|c|c|c|c|c|c|}
\hline \multirow[b]{2}{*}{ Sample } & \multicolumn{6}{|c|}{$R^{2}$ values } \\
\hline & Fluoride & Chloride & Nitrite & Bromide & Sulfate & Nitrate \\
\hline 0.200 ppm Fluoride & 0.9994 & 0.9905 & 0.9575 & 0.9308 & 0.8959 & 0.7140 \\
\hline 0.280 ppm Fluoride & 1.0000 & 0.9893 & 0.9543 & 0.9223 & 0.8858 & 0.6933 \\
\hline 0.660 ppm Fluoride & 0.9988 & 0.9832 & 0.9436 & 0.9009 & 0.8591 & 0.6316 \\
\hline 2.00 ppm Fluoride & 1.0000 & 0.9826 & 0.9381 & 0.8975 & 0.8528 & 0.6199 \\
\hline 2.85 ppm Fluoride & 0.9999 & 0.9798 & 0.9327 & 0.8923 & 0.8476 & 0.6293 \\
\hline 6.66 ppm Fluoride & 1.0000 & 0.9850 & 0.9344 & 0.9057 & 0.8631 & 0.6354 \\
\hline 20.0 ppm Fluoride & 1.0000 & 0.9922 & 0.9460 & 0.9485 & 0.9159 & 0.6782 \\
\hline 1.00 ppm Chloride & 0.9856 & 1.0000 & 0.9853 & 0.9586 & 0.9256 & 0.7181 \\
\hline 1.43 ppm Chloride & 0.9866 & 0.9999 & 0.9835 & 0.9558 & 0.9218 & 0.7106 \\
\hline 3.33 ppm Chloride & 0.9797 & 1.0000 & 0.9831 & 0.9585 & 0.9255 & 0.7115 \\
\hline 10.0 ppm Chloride & 0.9856 & 1.0000 & 0.9779 & 0.9608 & 0.9292 & 0.7076 \\
\hline 14.3 ppm Chloride & 0.9972 & 1.0000 & 0.9738 & 0.9608 & 0.9288 & 0.7053 \\
\hline 33.3 ppm Chloride & 0.6515 & 1.0000 & 0.9554 & 0.9635 & 0.9164 & 0.7173 \\
\hline 100 ppm Chloride & 0.0065 & 1.0000 & 0.4669 & 0.0159 & 0.5534 & 0.0200 \\
\hline 1.00 ppm Nitrite & 0.9588 & 0.9826 & 1.0000 & 0.9900 & 0.9715 & 0.7888 \\
\hline 1.43 ppm Nitrite & 0.9547 & 0.9821 & 1.0000 & 0.9915 & 0.9736 & 0.7908 \\
\hline 3.33 ppm Nitrite & 0.9455 & 0.9803 & 1.0000 & 0.9925 & 0.9756 & 0.7918 \\
\hline 10.0 ppm Nitrite & 0.9294 & 0.9784 & 1.0000 & 0.9946 & 0.9795 & 0.7870 \\
\hline 14.3 ppm Nitrite & 0.9319 & 0.9755 & 1.0000 & 0.9964 & 0.9829 & 0.7887 \\
\hline 33.3 ppm Nitrite & 0.9805 & 0.9636 & 1.0000 & 0.9993 & 0.9893 & 0.8026 \\
\hline 100 ppm Nitrite & 0.1295 & 0.9495 & 1.0000 & 0.9606 & 0.9725 & 0.8754 \\
\hline 1.00 ppm Bromide & 0.9239 & 0.9578 & 0.9900 & 1.0000 & 0.9946 & 0.8553 \\
\hline 1.43 ppm Bromide & 0.9210 & 0.9580 & 0.9907 & 1.0000 & 0.9946 & 0.8554 \\
\hline 3.33 ppm Bromide & 0.9132 & 0.9582 & 0.9910 & 1.0000 & 0.9944 & 0.8509 \\
\hline 10.0 ppm Bromide & 0.8931 & 0.9588 & 0.9927 & 1.0000 & 0.9945 & 0.8461 \\
\hline 14.3 ppm Bromide & 0.8938 & 0.9598 & 0.9947 & 1.0000 & 0.9944 & 0.8412 \\
\hline 33.3 ppm Bromide & 0.9497 & 0.9597 & 0.9979 & 1.0000 & 0.9935 & 0.8328 \\
\hline 100 ppm Bromide & 0.0258 & 0.9634 & 0.9934 & 1.0000 & 0.9856 & 0.8151 \\
\hline 1.00 ppm Nitrate & 0.8871 & 0.9234 & 0.9724 & 0.9942 & 1.0000 & 0.9005 \\
\hline 1.43 ppm Nitrate & 0.8847 & 0.9247 & 0.9744 & 0.9945 & 1.0000 & 0.8970 \\
\hline 3.33 ppm Nitrate & 0.8739 & 0.9257 & 0.9762 & 0.9946 & 1.0000 & 0.8933 \\
\hline 10.0 ppm Nitrate & 0.8478 & 0.9244 & 0.9790 & 0.9940 & 1.0000 & 0.8861 \\
\hline 14.3 ppm Nitrate & 0.8554 & 0.9259 & 0.9823 & 0.9942 & 1.0000 & 0.8790 \\
\hline 33.3 ppm Nitrate & 0.9408 & 0.9215 & 0.9888 & 0.9924 & 1.0000 & 0.8626 \\
\hline 100 ppm Nitrate & 0.0225 & 0.9152 & 0.9653 & 0.9787 & 1.0000 & 0.8207 \\
\hline 1.00 ppm Sulfate & 0.6898 & 0.7099 & 0.8035 & 0.8494 & 0.8954 & 0.9998 \\
\hline 1.43 ppm Sulfate & 0.6774 & 0.7061 & 0.8018 & 0.8464 & 0.8929 & 1.0000 \\
\hline 3.33 ppm Sulfate & 0.6467 & 0.6990 & 0.7969 & 0.8396 & 0.8870 & 0.9998 \\
\hline 10.0 ppm Sulfate & 0.6072 & 0.6890 & 0.7944 & 0.8298 & 0.8776 & 1.0000 \\
\hline 14.3 ppm Sulfate & 0.6300 & 0.6887 & 0.7994 & 0.8295 & 0.8765 & 1.0000 \\
\hline 33.3 ppm Sulfate & 0.7766 & 0.6800 & 0.8198 & 0.8234 & 0.8648 & 1.0000 \\
\hline 100 ppm Sulfate & 0.4048 & 0.7221 & 0.8265 & 0.5537 & 0.7787 & 1.0000 \\
\hline
\end{tabular}


Table S7. Identity Correlation Matrix, Cation Separation on CS 16 Column

$R^{2}$ values

\begin{tabular}{|c|c|c|c|c|c|c|}
\hline Sample & Lithium & Sodium & Ammonium & Potassium & Magnesium & Calcium \\
\hline 0.100 ppm Lithium & 0.9995 & 0.9892 & 0.9385 & 0.8802 & 0.7350 & 0.6801 \\
\hline $0.300 \mathrm{ppm}$ Lithium & 1.0000 & 0.9915 & 0.9330 & 0.8641 & 0.6784 & 0.6073 \\
\hline 0.600 ppm Lithium & 0.9999 & 0.9920 & 0.9203 & 0.8625 & 0.6688 & 0.5944 \\
\hline 1.00 ppm Lithium & 0.9999 & 0.9919 & 0.9012 & 0.8605 & 0.6628 & 0.5882 \\
\hline 3.00 ppm Lithium & 0.9999 & 0.9910 & 0.8176 & 0.8573 & 0.6634 & 0.6001 \\
\hline 6.00 ppm Lithium & 1.0000 & 0.9910 & 0.7863 & 0.8530 & 0.7034 & 0.6616 \\
\hline 10.0 ppm Lithium & 1.0000 & 0.9911 & 0.8428 & 0.8250 & 0.7540 & 0.7338 \\
\hline 30.0 ppm Lithium & 1.0000 & 0.9934 & 0.1304 & 0.6116 & 0.6583 & 0.6122 \\
\hline 60.0 ppm Lithium & 1.0000 & 0.9106 & 0.1431 & 0.0067 & 0.1801 & 0.3168 \\
\hline 100 ppm Lithium & 1.0000 & 0.0118 & 0.0317 & 0.0728 & 0.0395 & 0.3478 \\
\hline $0.100 \mathrm{ppm}$ Sodium & 0.9939 & 0.9993 & 0.9755 & 0.9209 & 0.8271 & 0.7826 \\
\hline $0.300 \mathrm{ppm}$ Sodium & 0.9914 & 0.9999 & 0.9758 & 0.9156 & 0.7663 & 0.6990 \\
\hline 0.600 ppm Sodium & 0.9896 & 0.9999 & 0.9737 & 0.9151 & 0.7450 & 0.6673 \\
\hline 1.00 ppm Sodium & 0.9888 & 0.9999 & 0.9693 & 0.9153 & 0.7393 & 0.6586 \\
\hline 3.00 ppm Sodium & 0.9895 & 0.9998 & 0.9386 & 0.9095 & 0.7230 & 0.6398 \\
\hline 6.00 ppm Sodium & 0.9907 & 0.9999 & 0.8934 & 0.9031 & 0.7248 & 0.6515 \\
\hline 10.0 ppm Sodium & 0.9914 & 1.0000 & 0.8567 & 0.8936 & 0.7335 & 0.6727 \\
\hline 30.0 ppm Sodium & 0.9927 & 0.9999 & 0.8710 & 0.8454 & 0.7865 & 0.7668 \\
\hline 60.0 ppm Sodium & 0.9929 & 1.0000 & 0.0060 & 0.7773 & 0.7703 & 0.7275 \\
\hline 100 ppm Sodium & 0.9898 & 1.0000 & 0.0925 & 0.0706 & 0.6216 & 0.6327 \\
\hline $0.100 \mathrm{ppm}$ Ammonium & 0.9638 & 0.9898 & 0.9977 & 0.9435 & 0.8621 & 0.8138 \\
\hline $0.300 \mathrm{ppm}$ Ammonium & 0.9454 & 0.9792 & 0.9998 & 0.9477 & 0.8121 & 0.7245 \\
\hline 0.600 ppm Ammonium & 0.9278 & 0.9671 & 0.9998 & 0.9507 & 0.8194 & 0.7226 \\
\hline 1.00 ppm Ammonium & 0.9133 & 0.9565 & 0.9993 & 0.9534 & 0.8225 & 0.7122 \\
\hline 3.00 ppm Ammonium & 0.8759 & 0.9271 & 0.9992 & 0.9573 & 0.8552 & 0.7381 \\
\hline $6.00 \mathrm{ppm}$ Ammonium & 0.8512 & 0.9068 & 0.9999 & 0.9582 & 0.8777 & 0.7628 \\
\hline 10.0 ppm Ammonium & 0.8324 & 0.8906 & 0.9999 & 0.9567 & 0.8918 & 0.7783 \\
\hline 30.0 ppm Ammonium & 0.7941 & 0.8562 & 0.9995 & 0.9558 & 0.9118 & 0.7955 \\
\hline 60.0 ppm Ammonium & 0.7685 & 0.8312 & 0.9997 & 0.9584 & 0.9178 & 0.7970 \\
\hline 100 ppm Ammonium & 0.7504 & 0.8114 & 1.0000 & 0.9638 & 0.9187 & 0.7963 \\
\hline 0.100 ppm Potassium & 0.8528 & 0.9071 & 0.9516 & 0.9993 & 0.9167 & 0.8467 \\
\hline 0.300 ppm Potassium & 0.8483 & 0.9029 & 0.9505 & 0.9997 & 0.9012 & 0.8112 \\
\hline 0.600 ppm Potassium & 0.8406 & 0.8958 & 0.9482 & 0.9999 & 0.8978 & 0.7966 \\
\hline 1.00 ppm Potassium & 0.8353 & 0.8905 & 0.9457 & 0.9999 & 0.8979 & 0.7970 \\
\hline 3.00 ppm Potassium & 0.8270 & 0.8818 & 0.9501 & 0.9995 & 0.8964 & 0.7943 \\
\hline $6.00 \mathrm{ppm}$ Potassium & 0.8239 & 0.8783 & 0.9530 & 0.9996 & 0.8909 & 0.7926 \\
\hline 10.0 ppm Potassium & 0.8193 & 0.8741 & 0.9515 & 0.9999 & 0.8874 & 0.7907 \\
\hline 30.0 ppm Potassium & 0.7914 & 0.8491 & 0.9594 & 0.9995 & 0.8803 & 0.7867 \\
\hline $60.0 \mathrm{ppm}$ Potassium & 0.7522 & 0.8077 & 0.8888 & 0.9999 & 0.8813 & 0.7945 \\
\hline 100 ppm Potassium & 0.7120 & 0.7566 & 0.2760 & 1.0000 & 0.8871 & 0.8375 \\
\hline 0.100 ppm Magnesium & 0.6462 & 0.7104 & 0.7941 & 0.9162 & 0.9995 & 0.9676 \\
\hline $0.300 \mathrm{ppm}$ Magnesium & 0.6391 & 0.7038 & 0.7945 & 0.9103 & 0.9999 & 0.9687 \\
\hline 0.600 ppm Magnesium & 0.6300 & 0.6939 & 0.7930 & 0.9032 & 1.0000 & 0.9669 \\
\hline 1.00 ppm Magnesium & 0.6264 & 0.6894 & 0.7989 & 0.9008 & 0.9999 & 0.9673 \\
\hline 3.00 ppm Magnesium & 0.6170 & 0.6804 & 0.8360 & 0.8962 & 0.9998 & 0.9683 \\
\hline 6.00 ppm Magnesium & 0.6110 & 0.6758 & 0.8768 & 0.8948 & 0.9999 & 0.9673 \\
\hline 10.0 ppm Magnesium & 0.6048 & 0.6701 & 0.9069 & 0.8957 & 1.0000 & 0.9617 \\
\hline 30.0 ppm Magnesium & 0.5592 & 0.6212 & 0.9198 & 0.8909 & 1.0000 & 0.9497 \\
\hline 60.0 ppm Magnesium & 0.5042 & 0.5469 & 0.6963 & 0.8821 & 1.0000 & 0.9553 \\
\hline 100 ppm Magnesium & 0.4629 & 0.4859 & 0.4013 & 0.8234 & 1.0000 & 0.9553 \\
\hline 0.100 ppm Calcium & 0.5203 & 0.5791 & 0.6645 & 0.8047 & 0.9733 & 0.9997 \\
\hline 0.300 ppm Calcium & 0.5127 & 0.5704 & 0.6627 & 0.7974 & 0.9695 & 0.9999 \\
\hline 0.600 ppm Calcium & 0.5061 & 0.5628 & 0.6655 & 0.7909 & 0.9655 & 1.0000 \\
\hline 1.00 ppm Calcium & 0.5020 & 0.5583 & 0.6758 & 0.7874 & 0.9631 & 0.9999 \\
\hline 3.00 ppm Calcium & 0.4948 & 0.5514 & 0.7293 & 0.7876 & 0.9618 & 0.9999 \\
\hline 6.00 ppm Calcium & 0.4769 & 0.5344 & 0.7674 & 0.7807 & 0.9594 & 0.9999 \\
\hline 10.0 ppm Calcium & 0.4597 & 0.5168 & 0.7880 & 0.7748 & 0.9549 & 0.9998 \\
\hline 30.0 ppm Calcium & 0.3974 & 0.4438 & 0.7884 & 0.7451 & 0.9349 & 0.9998 \\
\hline 60.0 ppm Calcium & 0.3490 & 0.3688 & 0.4699 & 0.7294 & 0.9231 & 1.0000 \\
\hline 100 ppm Calcium & 0.3202 & 0.3554 & 0.3546 & 0.5123 & 0.9241 & 1.0000 \\
\hline
\end{tabular}




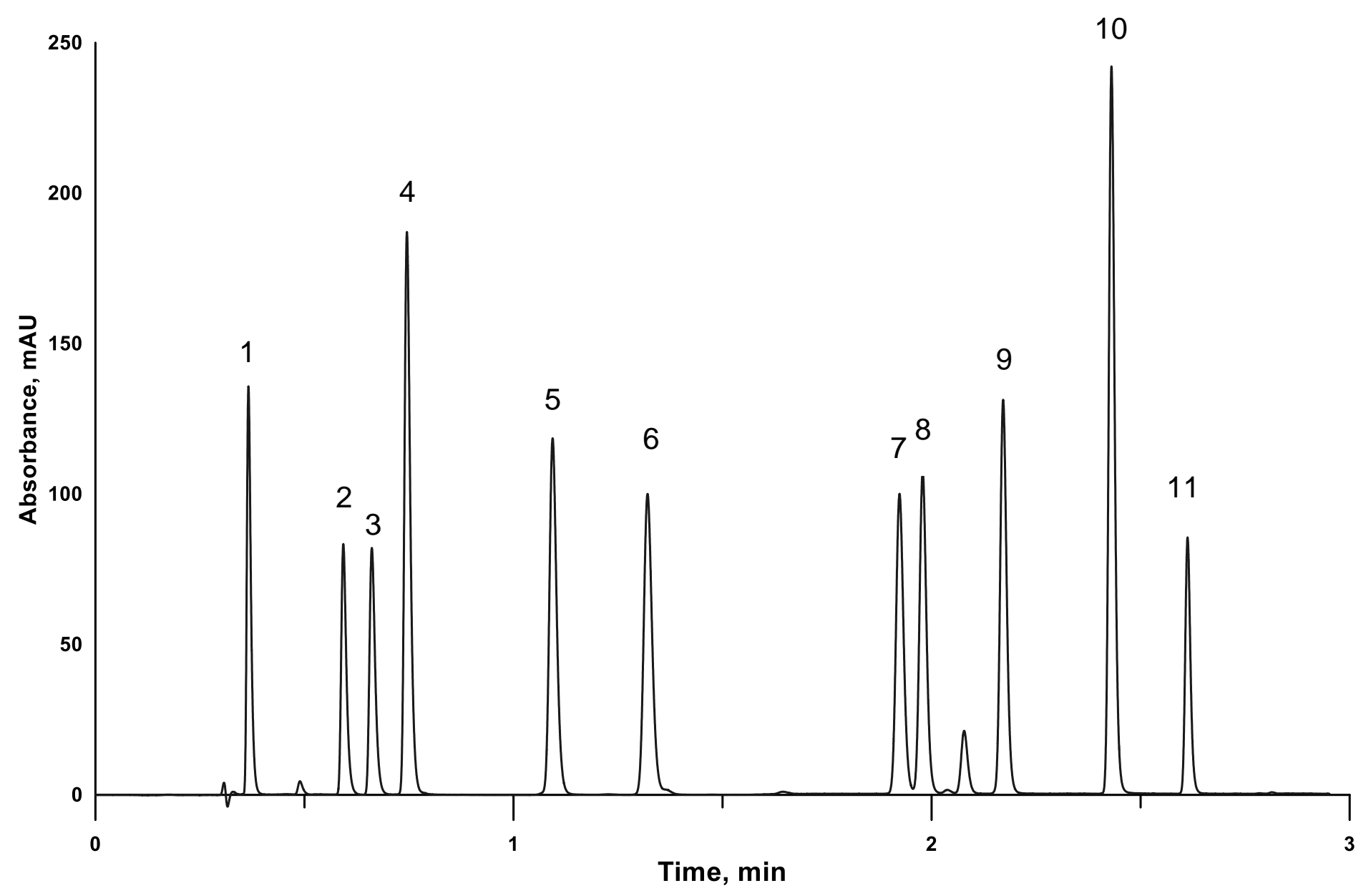

Figure S11. Separation of phenylurea pesticides (all concentrations $20 \mu \mathrm{g} / \mathrm{mL}$ ). See main text for experimental conditions. Peak identities: 1-Tebuthiuron, 2-Thidiazuron, 3-Monuron, 4-Fluometuron, 5-Diuron, 6-Propanil, 7-Siduron A, 8-Siduron B, 9-Liunuron, 10-Carbazole, 11-Diflubenzuron 
Table S8. Phenylurea Analysis (RPLC-UV, Figure S11) $r^{2}$ Correlation Matrix

\begin{tabular}{|c|c|c|c|c|c|c|c|c|c|c|c|}
\hline \multirow[b]{2}{*}{ Standards } & \multicolumn{11}{|c|}{$\mathbf{R}^{2}$ values } \\
\hline & Tebuthiuron & Thidiazuron & Monuron & Fluometuron & Diuron & Propanil & Siduron $\mathbf{A}$ & Siduron B & Liunuron & Carbazole & Diflubenzuron \\
\hline Tebuthiuron - 1 ppm & 1.0000 & 0.9187 & 0.8374 & 0.8270 & 0.5716 & 0.4735 & 0.4852 & 0.5553 & 0.6847 & 0.7956 & 0.8965 \\
\hline Tebuthiuron - 20 ppm & 1.0000 & 0.9347 & 0.8537 & 0.8215 & 0.5637 & 0.4669 & 0.4820 & 0.5702 & 0.6774 & 0.7886 & 0.8943 \\
\hline Thidiazuron - 1 ppm & 0.9272 & 1.0000 & 0.9829 & 0.9789 & 0.8366 & 0.7200 & 0.7763 & 0.8410 & 0.9080 & 0.9608 & 0.9883 \\
\hline Thidiazuron - $20 \mathrm{ppm}$ & 0.9302 & 1.0000 & 0.9843 & 0.9757 & 0.8259 & 0.7084 & 0.7649 & 0.8313 & 0.8987 & 0.9543 & 0.9878 \\
\hline Monuron - 1 ppm & 0.8555 & 0.9834 & 1.0000 & 0.9996 & 0.9208 & 0.8196 & 0.8736 & 0.9236 & 0.9634 & 0.9850 & 0.9753 \\
\hline Monuron - 20 ppm & 0.8669 & 0.9855 & 1.0000 & 0.9986 & 0.9106 & 0.8063 & 0.8650 & 0.9174 & 0.9588 & 0.9853 & 0.9845 \\
\hline Fluometuron - 1 ppm & 0.8444 & 0.9791 & 0.9996 & 1.0000 & 0.9302 & 0.8338 & 0.8860 & 0.9334 & 0.9701 & 0.9878 & 0.9728 \\
\hline Fluometuron - 20 ppm & 0.8323 & 0.8732 & 0.9242 & 1.0000 & 0.9270 & 0.8365 & 0.9017 & 0.9368 & 0.9728 & 0.9921 & 0.9627 \\
\hline Diuron - 1 ppm & 0.6997 & 0.8637 & 0.9325 & 0.9397 & 1.0000 & 0.9719 & 0.9931 & 0.9964 & 0.9846 & 0.9443 & 0.8683 \\
\hline Diuron - 20 ppm & 0.6973 & 0.8470 & 0.8650 & 0.9377 & 1.0000 & 0.9744 & 0.9974 & 0.9959 & 0.9853 & 0.9449 & 0.8893 \\
\hline Propanil - 1 ppm & 0.6309 & 0.7718 & 0.8498 & 0.8575 & 0.9729 & 1.0000 & 0.9875 & 0.9652 & 0.9297 & 0.8662 & 0.7761 \\
\hline Propanil - 20 ppm & 0.6323 & 0.7853 & 0.7833 & 0.8597 & 0.9753 & 1.0000 & 0.9857 & 0.9615 & 0.9302 & 0.8667 & 0.7977 \\
\hline Siduron A - 1 ppm & 0.5387 & 0.7584 & 0.8699 & 0.8813 & 0.9920 & 0.9872 & 1.0000 & 0.9908 & 0.9643 & 0.8990 & 0.7775 \\
\hline Siduron A - 20 ppm & 0.5520 & 0.8134 & 0.8202 & 0.8917 & 0.9955 & 0.9854 & 1.0000 & 0.9915 & 0.9684 & 0.9050 & 0.8085 \\
\hline Siduron B - 1 ppm & 0.6399 & 0.8425 & 0.9265 & 0.9356 & 0.9954 & 0.9617 & 0.9897 & 1.0000 & 0.9913 & 0.9516 & 0.8600 \\
\hline Siduron B - 20 ppm & 0.6464 & 0.8616 & 0.8871 & 0.9388 & 0.9960 & 0.9618 & 0.9912 & 1.0000 & 0.9927 & 0.9543 & 0.8813 \\
\hline Liunuron - 1 ppm & 0.7750 & 0.9207 & 0.9682 & 0.9738 & 0.9841 & 0.9247 & 0.9695 & 0.9922 & 1.0000 & 0.9857 & 0.9339 \\
\hline Liunuron - 20 ppm & 0.7717 & 0.8927 & 0.9281 & 0.9748 & 0.9849 & 0.9256 & 0.9724 & 0.9934 & 1.0000 & 0.9858 & 0.9440 \\
\hline Carbazole - 1 ppm & 0.8483 & 0.9652 & 0.9864 & 0.9889 & 0.9403 & 0.8484 & 0.9126 & 0.9553 & 0.9853 & 1.0000 & 0.9798 \\
\hline Carbazole - 20 ppm & 0.8276 & 0.5711 & 0.8362 & 0.9958 & 0.9425 & 0.8422 & 0.9180 & 0.9298 & 0.9859 & 1.0000 & 0.7708 \\
\hline Diflubenzuron - 1 ppm & 0.9115 & 0.9884 & 0.9751 & 0.9730 & 0.8461 & 0.7323 & 0.7988 & 0.8620 & 0.9251 & 0.9776 & 1.0000 \\
\hline Diflubenzuron - 20 ppm & 0.9107 & 0.9883 & 0.9830 & 0.9766 & 0.8510 & 0.7380 & 0.8046 & 0.8683 & 0.9280 & 0.9789 & 1.0000 \\
\hline
\end{tabular}

The highlighting is based on a $r^{2}$ threshold value of 0.9990 . With this threshold there is one false confirmation of Monuron as Fluometuron and vice-versa 